\title{
Optimizing radiation dose and fractionation for the definitive treatment of locally advanced non-small cell lung cancer
}

\author{
Michael C. Roach, Jeffrey D. Bradley, Cliff G. Robinson \\ Department of Radiation Oncology, Washington University School of Medicine, Saint Louis, MO, USA \\ Contributions: (I) Conception and design: MC Roach; (II) Administrative support: None; (III) Provision of study materials or patients: MC Roach; \\ (IV) Collection and assembly of data: MC Roach; (V) Data analysis and interpretation: All authors; (VI) Manuscript writing: All authors; (VII) Final \\ approval of manuscript: All authors. \\ Correspondence to: Michael C. Roach, MD. 660 S Euclid Ave, Campus Box 8224, Saint Louis, Missouri 63110, USA. Email: roachm@wustl.edu.
}

\begin{abstract}
Radiation therapy is the foundation for treatment of locally advanced non-small cell lung cancer (NSCLC), a disease that is often inoperable and has limited long term survival. Local control of disease is strongly linked to patient survival and continues to be problematic despite continued attempts at changing the dose and fractionation of radiation delivered. Technological advancements such as 4-dimensional computed tomography (CT) based planning, positron emission tomography (PET) based target delineation, and daily image guidance have allowed for ever more accurate and conformal treatments. A limit to dose escalation with conventional fractions of 2 Gy once per day appears to have been reached at 60 Gy in the randomized trial Radiation Therapy Oncology Group (RTOG) 0617. Higher doses were surprisingly associated with worse overall survival. Approaches other than conventional dose escalation have been explored to better control disease including accelerating treatment to limit tumor repopulation both with hyperfractionation and its multiple small ( $<2$ Gy) fractions each day and with hypofractionation and its single larger (>2 Gy) fraction each day. These accelerated regimens are increasingly being used with concurrent chemotherapy, and multiple institutions have reported it as tolerable. Tailoring treatment to individual patient disease and normal anatomic characteristics has been explored with isotoxic dose escalation up to the tolerance of organs at risk, with both hyperfractionation and hypofractionation. Metabolic imaging during and after treatment is increasingly being used to boost doses to residual disease. Boost doses have included moderate hypofractionation of 2-4 Gy, and more recently extreme hypofractionation with stereotactic body radiation therapy (SBRT). In spite of all these changes in dose and fractionation, lung and cardiovascular toxicity remain obstacles that limit disease control and patient survival.
\end{abstract}

Keywords: Radiotherapy; carcinoma; non-small cell lung; dose fractionation

Submitted Sep 30, 2017. Accepted for publication Jan 23, 2018.

doi: $10.21037 /$ jtd.2018.01.153

View this article at: http://dx.doi.org/10.21037/jtd.2018.01.153

\section{Introduction}

Lung cancer has long been the most common cause of cancer death worldwide, claiming 1.7 million lives annually (1). Non-small cell is the most common type of lung cancer diagnosed, and approximately $25 \%$ of patients will present with locally advanced disease (2). Locally advanced disease is typically defined as American Joint Committee on
Cancer stage III, with unresectable primary tumors (T4) or involvement of lymph nodes in the mediastinum (N2) or supraclavicular fossa (N3) (3). While locally advanced disease is curable, patient outcomes unfortunately remain poor despite both radiation therapy, chemotherapy, and now immunotherapy having improved over time.

The foundation of treatment for locally advanced disease is radiation therapy. Radiation is usually combined with 
concurrent platinum-based chemotherapy, as several trials and a meta-analysis have shown improved overall survival and local control with concurrent rather than sequential treatment (4). Control of disease with concurrent therapy remains challenging, however, with 2-year progression free survival at just $20-30 \%$. Though the development of distant metastases is most common with approximately $50 \%$ of patients experiencing this at 2 years, $30-40 \%$ of patients also develop local failure in 2 years (5). Resection after concurrent chemoradiation therapy show that this combination of radiation and chemotherapy only leads to complete response in $35-45 \%$ of patients $(6,7)$. This review will focus on escalation of the total radiation dose used for treatment, as well as changes to dose and fractionation that are being explored in attempts to improve local control and outcomes in locally advanced disease.

\section{Conventional fractionation}

Radiation therapy for locally advanced non-small cell lung cancer (NSCLC) is most commonly given in fractions of 1.8 to 2.0 Gy once a day for five days a week with external beams. Radiation Therapy Oncology Group (RTOG) 7301 established a dose of 60 Gy as the standard, after escalating the total dose from $40 \mathrm{~Gy}$ in a split course, then to continuous delivery from 40 , to 50 , and finally to $60 \mathrm{~Gy}$, with the higher doses having higher intrathoracic tumor control rates (8). It should be noted that this dose escalation was done in the two dimensional era when plans were made on based upon $\mathrm{X}$-rays. This was before computed tomography (CT) scanners were used in the clinic. Since then, advances in radiation treatment have included three dimensional CT-based conformal treatment planning, the use of positron emission tomography (PET) for more accurate staging and targeting, and accounting for tumor motion during treatment.

Traditionally, external beam radiation therapy for locally advanced NSCLC targeted the primary tumor as well as the ipsilateral hilar and mediastinal nodal stations, and sometimes even the supraclavicular fossa. This was done even without evidence of involvement in these regions and is known as elective nodal irradiation. This was done to control any microscopic disease in areas felt to be high risk for regional metastases. The concern with elective nodal irradiation, however, is that it will limit dose escalation as the larger volume irradiated will lead to otherwise avoidable pulmonary, cardiovascular, and esophageal toxicity. Three randomized trials on its use and several cohorts have been reported. A meta-analysis of these studies shows the overall incidence of elective nodal failure was $5.5 \%$ without elective nodal irradiation and $3.4 \%$ with it $(\mathrm{P}=0.64$ assuming a fixedeffects mode) (9). Given this lack of a significant difference, radiation is now most often prescribed only to gross disease that is enlarged on CT or hypermetabolic on PET.

\section{Conventional dose escalation}

In attempts to improve local control, radiation dose escalation with conventional fractionation and concurrent chemotherapy was performed by several groups in phase I and II trials. RTOG 0117 escalated to 75.25 Gy in 2.15 Gy daily fractions prescribed to the isocenter. Three of the eight patients who received 75.25 Gy developed dose-limiting pulmonary toxicity, one of which was fatal. The maximum tolerated radiation dose was therefore determined to be 74 Gy in 2 Gy fractions (10). North Central Cancer Treatment Group (NCCTG) 0028 escalated from 70 Gy up in 4 Gy increments. Similar to RTOG 0117, the maximum tolerated dose was also determined to be $74 \mathrm{~Gy}$. Two of four patients experienced dose limiting pneumonitis at 78 Gy (11). The University of North Carolina first escalated from 60 to $74 \mathrm{~Gy}$ both with limited elective nodal radiation and with induction and concurrent chemotherapy, then later from 74 to 90 Gy showing grade 3 or greater late complications in $22 \%$ of patients (12). Cancer and Leukemia Group B (CALGB) 30105 was a phase II trial treating at $74 \mathrm{~Gy}$ and randomizing between gemcitabine and paclitaxel used in both induction and concurrent treatment (13). Aside from the gemcitabine arm of this trial which closed early due to high toxicity, most of these trials showed a median survival of around 2 years.

These trials then led to the multi-institutional randomized controlled phase III trial RTOG 0617. In this $2 \times 2$ factorial design trial, patients with stage III NSCLC all received weekly carboplatin and paclitaxel chemotherapy with concurrent radiation in 2 Gy per once daily fraction followed by two cycles of consolidative chemotherapy after the completion of radiation. Patients were randomized to receive either 60 or $74 \mathrm{~Gy}$ and to receive this with or without cetuximab. At the first interim analysis, the monitoring committee established that the trial had crossed the futility boundary with respect to the 74 Gy arm, and this high dose arm was closed. The trial continued accruing only at the 60 Gy arm. At the third interim analysis, it was established that the cetuximab arm had also crossed the futility boundary. 
The published results of 0617 showed significant increased rates of death in the 74 Gy arm, challenging the assumption established in the earlier phase I/II studies that radiotherapy dose escalation with conventional fractionation and concurrent chemotherapy will improve outcomes (14). The median survival was 28.7 months in the 60 Gy arm versus 19.5 months in the $74 \mathrm{~Gy}$ arm $(\mathrm{P}=0.0007$; HR 1.56, 95\% CI: 1.19-2.06) (5). There was a 37\% increased risk of local failure in the high dose arms $(\mathrm{P}=0.0319$, HR $1.37,95 \%$ CI: 0.99-1.89). There was no difference in severe (Common Terminology Criteria for Adverse Events grade $\geq 3$ ) toxic effects overall, in severe pulmonary events specifically, or in severe radiation pneumonitis between the radiation therapy dose groups. Severe esophagitis was more common in the high-dose group ( $21 \%$ vs. $16 \%, \mathrm{P}<0.0001)$. On multivariate analyses, factors predicting worse overall survival were maximum esophagitis grade, planning target volume size, heart dose, and radiation dose.

The poor survival with treatment to 74 Gy has been attributed to several causes. Treatment-related deaths were more common in the high-dose group than in the lowdose group (10 vs. 2), but this comparison did not reach statistical significance. Concurrent chemotherapy was more difficult to complete in the high-dose group than in the low-dose group. Rates of protocol non-compliance were greater in the high-dose arm, $26 \%$ vs. $17 \%(\mathrm{P}=0.02)$, as were treatment delays. Radiation therapy planning was more likely to be non-compliant in the high-dose group, and planning target volume coverage by the $95 \%$ isodose line was poorer in the high-dose group. Concerns that noncompliance in the high-dose groups produced these results led to analysis of overall survival only in those patients with radiation plans compliant with the protocol; nevertheless, overall survival was still better in the standard-dose groups than in the high-dose groups.

The fact that heart dose was a significant predictor of overall survival on the multivariate analysis of RTOG 0617 strongly suggests that it is not only dose to tumor that should be considered in future studies. While further analysis of RTOG 0617 is pending, three retrospective studies also suggest heart dose can predict overall survival and cardiac events. The largest with 322 patients identified higher doses as important for overall survival and generated a new and more conservative heart constraint of V $50<25 \%$, or letting no more than $25 \%$ of the heart exceed 50 Gy (15). Two smaller series of 125 and 112 patients focused on cardiac events and showed mean heart dose was important $(16,17)$. A secondary analysis of RTOG 0617 showed that patients treated at centers with high trial accrual, a potential surrogate for number of NSCLC patients treated annually, had better survival, lower esophageal and heart doses, and lower lethal events (18). Radiation associated cardiac toxicity after treatment of locally advanced NSCLC may occur earlier than historically understood, and thus heart doses should be minimized with any future attempts at dose escalation.

Likely due to PET staging and modern radiation therapy techniques, the 28.7-month median survival in the 60 Gy arm was longer than that seen in previous studies, was better than anticipated, and set a new benchmark for patients with locally advanced NSCLC receiving concurrent chemotherapy and radiation therapy. Consequently, current trials in the United States such as RTOG 1306 and National Research Group (NRG) L001 have adopted 60 Gy as the standard, as well as the American Society for Radiation Oncology (ASTRO) in its guidelines (19). The National Comprehensive Cancer Network (NCCN) guidelines in the United States now suggest definitive radiation should be 60 to 70 Gy (20). The European Society for Medical Oncology guidelines state that "dose in excess of 66 Gy is not recommended outside trials" (21). The Cancer Council of Australia (CCA) says that radiation dose "should be at least 60 Gy assuming that dose-volume constraints on organs at risk are met" and that "74 Gy is not better than $60 \mathrm{~Gy}$ and may be potentially harmful" (22). Many other national guidelines, such as China's from 2015 (23), do not make explicit dose recommendations yet.

\section{Hyperfractionation}

Not all patients will be felt fit enough to tolerate concurrent treatment with chemotherapy, however, and many of those same national guidelines recommend accelerated hyperfractionation in such patients. The National Institute for Health and Care Excellence (NICE) and CCA both recommend continuous hyperfractionated accelerated radiation therapy (CHART) when only radiation therapy will be used as treatment. Acceleration refers to finishing treatment more rapidly than is done conventionally. Hyperfractionation refers to dividing treatment into smaller doses more than once a day. The CHART trial compared 36 fractions of 1.5 Gy given three times per day to a total dose of 54 Gy in 12 consecutive days with 30 fractions of 2 Gy once a day to a total dose of 60 Gy in 6 weeks. CHART improved 2-year overall survival from 
$20 \%$ to $29 \%$ ( $\mathrm{P}=0.008)$. The majority of patients $(81 \%)$ had squamous cell cancer, and in such patients, 2-year overall survival improved from $20 \%$ to $33 \%(\mathrm{P}=0.0007)$ (24). This regimen has never been directly compared to conventional chemoradiation in a randomized trial, however. An individual patient data meta-analysis showed that hyperfractionation improved overall survival by $2.5 \%$ at 5 years without improving progression free survival when compared to conventionally fractionated radiation alone. This came at the risk of worse acute esophageal toxicity (odds ratio of 2.44 in NSCLC) (25). The theory is that prolongation of treatment results in increased tumor cell repopulation (26), and has been proposed as one reason why the high dose arm of RTOG 0617 failed.

However, the CHART regimen is inconvenient as many centers are closed on weekends, and it often requires that the patients be hospitalized. The randomized phase III CHARTWEL (continuous hyperfractionated accelerated radiotherapy weekend less) looked at 60 Gy in 40 fractions over 2.5 weeks versus 66 Gy in 33 fractions over 6.5 weeks. In contrast to CHART, CHARTWEL showed no difference in overall survival and local tumor control. Exploratory analysis suggested trends for improved local control with hyperfractionation for higher stages of disease and after neoadjuvant chemotherapy. Histology did not affect control, and it should be noted that $58 \%$ of patients had squamous cell cancers, in contrast to the $81 \%$ in CHART where hyperfractionation showed the most promise in this histology. Another potential explanation for the lack of difference in overall survival was the fact that total radiation doses were $10 \%$ higher in both arms of CHARTWEL when compared to CHART (27).

Hyperfractionated radiation with concurrent chemotherapy has also been compared to the combination with conventional radiation. A $2 \times 2$ trial randomizing 60 Gy in 30 fractions in either 6 weeks once a day or 3 weeks twice a day with or without concurrent carboplatin showed no statistically significant difference in local recurrence or survival in all arms (28). RTOG 9410 showed that concurrent chemotherapy with hyperfractionated radiation to $69.6 \mathrm{~Gy}$ had statistically similar 5-year overall survival when compared to conventional chemoradiation to $63 \mathrm{~Gy}$, $13 \%$ vs. $16 \%(\mathrm{P}=0.46)$. Grade 3 esophagitis was statistically worse at $45 \%$ with hyperfractionation, double that of $22 \%$ with conventional fractionation $(\mathrm{P}<0.001)(29)$. When coupled with the logistical issues of delivering multiple fractions per day and patient inconvenience, for patients felt fit enough for concurrent treatment, conventional fractionation became standard in future trials.

\section{Hypofractionation}

Most recent studies have investigated accelerating treatment with hypofractionation with larger radiation doses delivered once per day, rather than with hyperfractionation and its smaller doses delivered more than once per day. Concerns about serious toxicity delayed exploration of hypofractionation until technology such as CT based planning, daily image guidance, and gating became widespread. Most reports have been for modest hypofractionation of 2-3 Gy per fraction. In patients receiving radiation alone, three studies comparing standard fractionation to hypofractionation did not report any significant differences in toxicity or disease outcomes (30-32). In a review of 22 studies of hypofractionated radiation alone, Kaster $e t$ al. found the weighted mean acute toxicity in the esophagus and lung being $1.9 \%$ and $1.2 \%$ respectively. Late toxicity was also low at $1.4 \%$ and $6.9 \%$. Two-year overall survival ranged from $18 \%$ to $42 \%$. There was a moderate linear relationship between biologically effective lesional dose (BED10): for every 1 Gy increase in BED10, there was an absolute overall survival benefit ranging from $0.36 \%$ to $0.70 \%$ (33). This is similar to the results found by Machtay et al. with conventional radiation where a 1 Gy increase in BED resulted in a $4 \%$ relative improvement in survival (14), with in the context of $15 \%$ long-term survival, is an absolute improvement of $0.6 \%$.

Hypofractionation may allow for better outcomes by increasing BED without lengthening treatment time and thereby preventing cancer cell repopulation. Studies of modest hypofractionation with concurrent chemotherapy are fewer and mostly single arm, single institution studies. In the same systemic review by Kaster et al., 15 studies of hypofractionated radiation therapy with concurrent chemotherapy were found. The weighted mean acute toxicity in the esophagus and lung was $14.9 \%$ and $7.9 \%$ respectively, and for weighted mean late toxicity in the esophagus and lung, $6.6 \%$ and $12.2 \%$. In comparison, RTOG 0617 showed acute grade 3 esophagitis in $7 \%$ and grade 3 pneumonitis in $4 \%$ with late toxicity rates $<1 \%$ for both. Two-year overall survival with concurrent hypofractionation ranged from $24 \%$ to $58 \%$. The 2 -year overall survival in RTOG 0617's standard arm was 58\% (5). In the United Kingdom, 55 Gy in 20 fractions of 2.75 Gy is the most commonly used schedule, both with and without concurrent systemic therapy (34). 
More aggressive hypofractionation to 4 Gy per fraction has been also been explored in patients not felt fit for concurrent chemotherapy. MD Anderson used protons to escalate to 60 Gy cobalt-equivalent in 15 fractions in a phase 1 trial. At a median follow-up of 13 months, two of 25 patients experienced dose-limiting toxicities. One treated to 52.5 Gy cobalt-equivalent in 15 fractions developed a tracheoesophageal fistula after bevacizumab was delivered for recurrent disease. The second developed "possible" grade 3 radiation pneumonitis (35). This regimen with protons is currently being explored with concurrent chemotherapy in a phase I study at our institution (NCT02172846).

As protons are not available at most centers, a phase 1 dose escalation study was later reported using photons at University of Texas Southwestern in patients who were not chemotherapy candidates. It enrolled 55 patients divided between 50, 55, and 60 Gy, all in 15 fractions. One patient developed grade 3 esophagitis, and 2 cases of grade 3 dyspnea were felt related to therapy. There was no association between fraction size and toxicity $(\mathrm{P}=0.24)$, and the median overall survival was 6 months at all dose levels $(\mathrm{P}=0.59)$ (36). This same group has presented in abstract form an interim analysis from a randomized phase III comparison of $60 \mathrm{~Gy}$ in 15 vs. 30 fractions of image-guided photon radiation therapy in patients with a Zubrod performance status of 2 or greater. Median overall survival for the 48 patients evaluable was 11.5 months with no statistical difference between conventional and hypofractionated radiation treatment arms. Two deaths from hypoxia with conventional radiation and 1 death with hypofractionated radiation were possibly related to treatment (37). Final results are pending, but the study authors feel the results could potentially change the paradigm of treatment for patients with locally advanced disease receiving radiation alone due to poor performance status.

\section{Adapted therapy}

Rather than prescribing the same fixed dose to all patients with locally advanced disease, isotoxic radiation therapy is a novel approach which allows for personalized treatment planning based on individual tumor and patient characteristics. This tailored approach is heavily based upon predefined organ at risk dose constraints. Treatment plans are designed to give the maximum BED achievable to the tumor target until the predefined dose constraints are reached. The increasing use of computer-based inverseplanned intensity modulated radiation therapy (IMRT) makes this approach especially feasible.

One such approach with hyperfractionation, individualized isotoxic accelerated radiotherapy (INDAR) has been used for over a decade in the Netherlands. In the first three weeks, 30 twice daily fractions of 1.5 Gy are delivered. Next, 2 Gy fractions once a day are delivered until a mean lung dose of 19 Gy is reached, with a total ranging between 54 to 69 Gy in 5.5 weeks. The equivalent conventional dose would be 72 Gy over 36 fractions. Long term results showed that with sequential chemotherapy and INDAR, the median survival was 23.6 months. For comparison, the use of sequential chemotherapy and conventional fractionation with the same group resulted in a lower median survival of only 17.5 months (38). The use of concurrent chemotherapy in a phase 1 study with INDAR showed no dose limiting toxicity (39).

Instead of hyperfractionation, other institutions have used isotoxic planning with hypofractionation. A single institution phase I trial used 25 fractions ranging from 2.28 to $3.42 \mathrm{~Gy}$ (a total of 57 to $85.5 \mathrm{~Gy}$ ) with IMRT. Total dose was escalated according to each patient's individual stratified risk for radiation pneumonitis. The maximum tolerated dose was predefined as the dose that theoretically would result in $\leq 20 \%$ risk of severe toxicity. Grade 4 to 5 toxicity was reached late in 6 of the 79 patients, and the maximum tolerated dose was defined as 63.25 Gy in 25 fractions of 2.53 Gy each. These severe toxicities were due to damage to the central and perihilar structures and corresponded to dose to the proximal bronchial tree (40).

RTOG 1106, which recently completed accrual, coupled the use of isotoxic hypofractionation with metabolic imaging to adapt chemoradiation treatment for locally advanced disease. The control arm was standard fractionation of 60 Gy in 30 fractions of 2 Gy each. In the experimental $\mathrm{arm}$, the first 21 fractions were 2.2 Gy each. The final nine fractions were delivered only to residual disease seen on a PET taken after fractions 18 or 19. Residual disease was defined as any sites with metabolic activity at least $150 \%$ that of the aortic arch. These final nine fractions could range from 2.2-3.8 Gy per fraction, corresponding to a range of 19.8 to $34.2 \mathrm{~Gy}$. The highest achievable dose was given while still respecting a mean lung dose of $20 \mathrm{~Gy}$. The theoretical maximum tumor dose was 80.4 Gy. Patients were randomized 2:1 into the experimental arm with stratification by stage, primary tumor size, and histology. The primary objective was local control, and results are 
pending.

RTOG 1106 was based on studies from University of Michigan which demonstrated that tumors decrease more in metabolic activity than size during treatment. Recently, this group reported on the results of a phase II study of conformal radiation individualized to a fixed risk of radiation-induced lung toxicity of $17.2 \%$ for grade 2 or greater pneumonitis. Dose to residual tumor was adaptively escalated to residual tumor on a mid-treatment PET up to a total dose of $86 \mathrm{~Gy}$ in 30 fractions. The median tumor dose delivered was 83 Gy over 30 fractions. The initial doses per fraction were 2.1 to 2.85 Gy initially over $18-24$ fractions, then 2.85 to 5.0 Gy for the adaptive phase after the midtreatment PET. Most patients (93\%) received concurrent carboplatin and paclitaxel with consolidation chemotherapy. With a median follow-up for surviving patients of 47 months, the 2-year rate of infield tumor control was $82 \%$ with a median overall survival of 25 months (41).

Rather than using mid-treatment imaging, other groups have looked at boosting any residual disease after the completion of conventional treatment with stereotactic body radiation therapy (SBRT). SBRT has proven very effective in early stage disease, especially in lesions far from major airways and the mediastinum (42). With SBRT, BEDs of at least 100 Gy total are typically delivered in 3-5 fractions with narrow margins. Four studies have looked at a SBRT boost to residual disease after conventional chemoradiation therapy to 50-60 Gy. In these small series with limited follow-up, local control at one year has been approximately $80 \%$ (43-46), comparable to that of RTOG 0617, which was a mixed group that contained patients who had a complete response. Of the 80 patients in these four studies of SBRT boosts, there have been $5(6.3 \%)$ lethal toxicities such as pulmonary hemorrhage, particularly with boosts to central disease near the main airways and mediastinum.

A multi-institutional trial has been proposed that would use a SBRT boost to metabolically active residual disease seen on PET-CT 2-4 weeks after chemoradiation treatment completion (47). Patients with a complete response would be observed. The benchmark for trial success would be that in patients with residual disease after conventional treatment, a SBRT boost would result in progressionfree survival of $20-30 \%$ at 2 years, matching that seen in RTOG 0617. Now with immunotherapy recently showing a progression free benefit when used after conventional chemoradiation (48), SBRT becomes even more attractive as a boost as it could theoretically better present tumor antigens and better serve as a potentiator of the patient's own immune system against their disease (49).

\section{Conclusions}

Locally advanced NSCLC remains a challenging disease with significant mortality and complications. Efforts to improve treatment outcomes have been only moderately successful, but the combination of hypofractionation with systemic therapy, individualized treatment adaptation, and stereotactic boosts in the era of immunotherapy offers the promise of further improvements.

\section{Acknowledgements}

None.

\section{Footnote}

Conflicts of Interest: The authors have no conflicts of interest to declare.

\section{References}

1. Global Burden of Disease Cancer Collaboration, Fitzmaurice C, Allen C, et al. Global, Regional, and National Cancer Incidence, Mortality, Years of Life Lost, Years Lived With Disability, and Disability-Adjusted Lifeyears for 32 Cancer Groups, 1990 to 2015: A Systematic Analysis for the Global Burden of Disease Study. JAMA Oncol 2017;3:524-48.

2. Morgensztern D, Ng S, Gao F, et al. Trends in stage distribution for patients with non-small cell lung cancer: a national cancer database survey. J Thorac Oncol 2010;5:29-33.

3. Edge S, Byrd DR, Compton CC, et al. AJCC Cancer Staging Manual. 7th Edition. Springer, November 2011.

4. Aupérin A, Le Pechoux C, Rolland E, et al. Meta-analysis of concomitant versus sequential radiochemotherapy in locally advanced non-small-cell lung cancer. J Clin Oncol 2010;28:2181-90.

5. Bradley JD, Paulus R, Komaki R, et al. Standard-dose versus high-dose conformal radiotherapy with concurrent and consolidation carboplatin plus paclitaxel with or without cetuximab for patients with stage IIIA or IIIB non-small-cell lung cancer (RTOG 0617): a randomised, two-by-two factorial phase 3 study. Lancet Oncol 2015;16:187-99. 
6. Cerfolio RJ, Bryant AS, Jones VL, et al. Pulmonary resection after concurrent chemotherapy and high dose (60Gy) radiation for non-small cell lung cancer is safe and may provide increased survival. Eur J Cardiothorac Surg 2009;35:718-23.

7. Edelman MJ, Suntharalingam M, Burrows W, et al. Phase I/II trial of hyperfractionated radiation and chemotherapy followed by surgery in stage III lung cancer. Ann Thorac Surg 2008;86:903-10.

8. Perez CA, Pajak TF, Rubin P, et al. Long-term observations of the patterns of failure in patients with unresectable non-oat cell carcinoma of the lung treated with definitive radiotherapy. Report by the Radiation Therapy Oncology Group. Cancer. 1987;59:1874-81.

9. Li R, Yu L, Lin S, et al. Involved field radiotherapy (IFRT) versus elective nodal irradiation (ENI) for locally advanced non-small cell lung cancer: a meta-analysis of incidence of elective nodal failure. Radiat Oncol 2016;11:124-31.

10. Bradley JD, Bae K, Graham MV, et al. Primary analysis of the phase II component of a phase I/II dose intensification sstudy using three-dimensional conformal radiation thera[y and concurrent chemotherapy for patient with inoperable non-small-cell lung cancer: RTOG 0117. J Clin Oncol 2010;28:2475-80.

11. Rosenman JG, Halle JS, Socinski MA, et al. Highdose conformal radiotherapy for treatment of stage IIIA/B non-small cell lung cancer: Technical issues and results of a phase I/II trial. Int J Radiat Oncol Biol Phys 2002;54:348-56.

12. Lee C. High-dose $3 \mathrm{D}$ chemoradiotherapy in stage III nonsmall cell lung cancer (NSCLC) at the University of North Carolina: Long-term follow up and late complications. Pro Amer Soc Clin Oncol 2006;24:7145.

13. Belani CP, Choy H, Bonomi P, et al. Combined chemoradiotherapy regimens of paclitaxel and carboplatin for locally advanced non-small-cell lung cancer: a randomized phase II locally advanced multi-modality protocol. J Clin Oncol 2005;23:5883-91.

14. Machtay M, Bae K, Movsas B, et al. Higher biologically effective dose of radiotherapy is associated with improved outcomes for locally advanced non-small cell lung carcinoma treated with chemoradiation: an analysis of the Radiation Therapy Oncology Group. Int J Radiat Oncol Biol Phys 2012;82:425-34.

15. Speirs CK, DeWees TA, Rehman S, et al. Heart dose is an independent dosimetric predictor of overall survival in locally advanced non-small cell lung cancer. J Thorac
Oncol 2017;12:293-301.

16. Dess RT, Sun Y, Matuszak MM, et al. Cardiac events after radiation therapy: combined analysis of prospective multicenter trials for locally advanced non-small-cell lung cancer. J Clin Oncol 2017;35:1395-402.

17. Wang K, Eblan MJ, Deal AM, et al. Cardiac toxicity after radiotherapy for stage III non-small-cell lung cancer: pooled analysis of dose-escalation trials delivering 70 to 90 Gy. J Clin Oncol 2017;35:1387-94.

18. Eaton BR, Pugh SL, Bradley JD, et al. Institutional enrollment and survival among NSCLC patients receiving chemoradiation: NRG Oncology Radiation Therapy Oncology Group (RTOG) 0617. J Natl Cancer Inst 2016;108. pii: djw034.

19. Rodrigues G, Choy H, Bradley J, et al. Definitive radiation therapy in locally advanced non-small cell lung cancer: Executive summary of an American Society for Radiation Oncology (ASTRO) evidencebased clinical practice guideline. Pract Radiat Oncol 2015;5:141-8.

20. National Comprehensive Cancer Network. Non-small cell lung cancer guidelines, version 8.2017: NSCL-C 8 of 10.

21. Postmus PE, Kerr KM, Oudkerk M, et al. Early and locally advanced non-small-cell lung cancer (NSCLC): ESMO clinical practice guidelines for diagnosis, treatment and follow-up. Ann Oncol 2017;28:iv1-21.

22. Lehman M, Cancer Council Australia Lung Cancer Guidelines Working Party. What is the optimal radiation dose and fractionation schedule for good performance status patients with inoperable stage III NSCLC undergoing curative therapy? Cited 2017 Sep 24. Available online: http://wiki.cancer.org.au/australiawiki/index. php?oldid=146538

23. Zhi XY, Yu JM, Shi YK, et al. Chinese guidelines on the diagnosis and treatment of primary lung cancer $(2015$ version). Cancer 2015;121:3165-81.

24. Saunders M, Dische S, Barrett A, et al. Continuous, hyperfractionated, accelerated radiotherapy (CHART) versus conventional radiotherapy in non-small cell lung cancer: mature data from the randomized multicenter trial. Radiother Oncol. 1999;52:137-48.

25. Mauguen A, Le Pechoux C, Saunders M, et al. Hyperfractioanted or accelerated radiotherapy in lung cancer: an individual patient data meta-analysis. J Clin Oncol 2012;30:2788-97.

26. Wang S, Liao Z, Wei X, et al. Analysis of clinical and dosimetric factors associated with treatment-related pneumonitis (TRP) in patients with non-small-cell lung 
cancer (NSCLC) treated with concurrent chemotherapy and three-dimensional conformal radiotherapy (3D-CRT). Int J Radiat Oncol Biol Phys 2006;66:1399-407.

27. Baumann M, Herrmann T, Koch R, et al. Final results of the randomized phase III CHARTWEL-trial (ARO 97-1) comparing hyperfractionated-accelerated versus conventionally fractionated radiotherapy in nonsmall cell lung cancer (NSCLC). Radiother Oncol 2011;100:76-85.

28. Ball D, Bishop J, Smith J, et al. A randomized phase III study of accelerated or standard fraction radiotherapy with or without concurrent carboplatin in inoperable non-small cell lung cancer: final report of an Australian multi-centre trial. Radiother Oncol 1999;52:129-36.

29. Curran WJ, Paulus R, Langer CJ, et al. Sequential vs concurrent chemoradiation for stage III non-small cell lung cancer: randomized phase III trial RTOG 9410. J Natl Cancer Inst 2011;103:1452-60.

30. Nguyen LN, Komaki R, Allen P, et al. Effectiveness of accelerated radiotherapy for patients with inoperable nonesmall cell lung cancer (NSCLC) and borderline prognostic factors without distant metastasis: a retrospective review. Int J Radiat Oncol Biol Phys 1999;44:1053-6.

31. Sun LM, Leung SW, Wang CJ, et al. Concomitant boost radiation therapy for inoperable nonesmall-cell lung cancer: preliminary report of a prospective randomized study. Int J Radiat Oncol Biol Phys 2000;47:413-8.

32. Amini A, Lin SH, Wei C, et al. Accelerated hypofractionated radiation therapy compared to conventionally fractionated radiation therapy for the treatment of inoperable nonesmall cell lung cancer. Radiat Oncol 2012;7:33

33. Kaster TS, Yaremko B, Palma D, et al. Radical-intent hypofractionated radiotherapy for locally advanced nonsmall-cell lung cancer: a systemic review of the literature. Clin Lung Cancer 2015;16:71-9.

34. Prewett SL, Aslam S, Williams MV, et al. The management of lung cancer: a UK survey of oncologists. Clin Oncol (R Coll Radiol) 2012;24:402-09.

35. Gomez DR, Gillin M, Liao Z, et al. Phase 1 study of dose escalation in hypofractionated proton beam therapy for non-small cell lung cancer. Int J Radiat Oncol Biol Phys 2013;86:665-70.

36. Westover KD, Loo BW Jr, Gerber DE, et al. Precision hypofractionated radiation therapy in poor performing patients with non-small cell lung cancer: phase 1 dose escalation trial. Int J Radiat Oncol Biol Phys 2015;93:72-81.
37. Iyengar P, Westover KD, Court LE, et al. A Phase III Randomized Study of Image Guided Conventional (60 Gy/30 fx) Versus Accelerated, Hypofractionated (60 Gy/15

fx) Radiation for Poor Performance Status Stage II and III NSCLC Patients-An Interim Analysis. Int J Radiat Oncol Biol Phys 2016;96:E451.

38. De Ruysscher D, van Baardwijk A, Steevens J, et al. ndividualised isotoxic accelerated radiotherapy and chemotherapy are associated with improved long-term survival of patients with stage III NSCLC: a prospective population-based study. Radiother Oncol 2012;102:228-33.

39. Dingemans AM, Bootsma G, van Baardwijk A, et al. A phase I study of concurrent individualized, isotoxic accelerated radiotherapy and cisplatin-vinorelbinecetuximab in patients with stage III non-small-cell lung cancer. J Thorac Oncol 2014;9:710-6.

40. Cannon DM, Mehta MP, Adkison JB, et al. Doselimiting toxicity after hypofractionated dose-escalated radiotherapy in non-small-cell lung cancer. J Clin Oncol 2013;31:4343-8.

41. Kong FM, Ten Haken RK, Schipper M, et al. Effect of Midtreatment PET/CT-Adapted Radiation Therapy With Concurrent Chemotherapy in Patients With Locally Advanced Non-Small-Cell Lung Cancer: A Phase 2 Clinical Trial. JAMA Oncol 2017;3:1358-65.

42. Roach MC, Videtic GM, Bradley JD. Treatment of peripheral non-small cell lung carcinoma with stereotactic body radiation therapy. J Thorac Oncol 2015;10:1261-7.

43. Feddock J, Arnold SM, Shelton BJ, et al. Stereotactic body radiation therapy can be used safely to boost residual disease in locally advanced non-small cell lung cancer: a prospective study. Int J Radiat Oncol Biol Phys 2013;85:1325-31.

44. Karam SD, Horne ZD, Hong RL, et al. Dose escalation with stereotactic body radiation therapy boost for locally advanced non small cell lung cancer. Radiat Oncol 2013;8:179.

45. Hepel JT, Leonard KL, Safran H, et al. Stereotactic body radiation therapy boost after concurrent chemoradiation for locally advanced non-small cell lung cancer: a phase 1 dose escalation study. Int J Radiat Oncol Biol Phys 2016;96:1021-7.

46. Trovo M, Minatel E, Durofil E, et al. Stereotactic body radiation therapy for reirradiation of persistent or recurrent non-small cell lung cancer. Int J Radiat Oncol Biol Phys 2014;88:1114-9.

47. Kalman NS, Weiss E, Walker PR, et al. Local 
Radiotherapy Intensification for Locally Advanced Nonsmall-cell Lung Cancer - A Call to Arms. Clin Lung Cancer 2018;19:17-26.

48. Antonia SJ, Villegas A, Daniel D, et al. Durvalumab after chemoradiotherapy in stage III non-small-cell lung cancer.
N Engl J Med 2017;377:1919-29.

49. Popp I, Grosu AL, Niedermann G, et al. Immune modulation by hypofractionated stereotactic radiation therapy: therapeutic implications. Radiother Oncol 2016;120:185-94.

Cite this article as: Roach MC, Bradley JD, Robinson CG. Optimizing radiation dose and fractionation for the definitive treatment of locally advanced non-small cell lung cancer. J Thorac Dis 2018;10(Suppl 21):S2465-S2473. doi: 10.21037/ jtd.2018.01.153 